\title{
CHOICE OF COURSE OF DEVIATION OF SHIP TAKING INTO ACCOUNT LAW OF DISTRIBUTING OF VECTOR'S ERROR
}

\section{ВЫБОР КУРСА УКЛОНЕНИЯ СУДНА С УЧЕТОМ ЗАКОНА РАСПРЕДЕЛЕНИЯ ВЕКТОРИАЛЬНОЙ ПОГРЕШНОСТИ}

\author{
A.V. Aleksichin, $P h D$, associate professor, A.I. Burmaka, $P h D$, associate \\ professor, A.R. Tsagikyan, assistant \\ А.В. Алексишин, к.т.н., дочент, А.И. Бурмака, к.т.н., доцент, А.Р. Цагикян, \\ ассистент \\ National University «Odessa Maritime Academy», Ukraine \\ Национальный университет «Одесская морская академия», Украина
}

\begin{abstract}
In the article dependence of circular safe region of ship is explored on the law of distributing of probability of position vector's error, which influences on the size of course of deviation of ship at his divergence with a dangerous target. It is presently offered more ten different forms of safe region of ship, although the safe region of ship of circular form is most often used. Therefore, this form of safe region from positions of dependence of its sizes from the closeness of law of distributing of position vector's error is considered in the article. Its radius is dipping out description of circular safe region of ship, which is evened maximum - possible distance of the shortest rapprochement, which, as shown in work, is the sum of two constituents of different on the nature. First component determined which depends on the sizes of ship and other determined factors. The second constituent is stochastic and it is simply determined by the law of distributing of probability of position vector's error, basic description of which there is matrix of its constituents.

The radius of stochastic constituent of circular safe region of ship gets out thus, that probability of hit of veritable place of ship in a region was near to 1 , on condition that the center of circular safe region coincides with the place of ship. In other words, a circular region with a center in position must cover the veritable place of ship with probability near to 1 . In work for implementation of this condition worked out an integral equation, in which in quality an unknown variable there is the radius of circular safe region of ship. So as in equalization an integral is certain, and a radius is in limits integration, integral equalization is regenerate to expression in the type of sum that allows defining the size of radius by the method of progressive approximations.

It is shown that the radius of circular safe region of ship depends on dispersions of constituents of position vector's error, which are determined by the scale parameter of law of distributing, and in the case of distributing of position vector's error on the mixed law of the first or second type on the size of radius the substantial parameter of law of distributing influences also.
\end{abstract}


Keywords: safety of navigation, safe region of ship, position vector's error.

\section{PEФЕРАT}

В статті досліджено залежність кругової безпечної області судна від закону розподілу вірогідності позиційної векторіальної похибки, що впливає на величину курсу ухилення судна при його розходженні з небезпечною ціллю. Нині запропоновано більш десяти різних форм безпечної області судна, хоча найбільш часто використовується безпечна область судна кругової форми. Тому в статті розглянута ця форма безпечної області з позицій залежності їі розмірів від двомірної щільності закону розподілу позиційної векторіальної похибки. Вичерпуючою характеристикою кругової безпечної області судна є іiї радіус, що дорівнює гранично - допустимій дистанції найкоротшого зближення, яка, як показано в роботі, являється сумою двох складових різних за своєю природою. Перша складова детермінована, яка залежить від розмірів судна та інших детермінованих чинників. Друга складова $є$ стохастичною і вона однозначно визначається законом розподілу вірогідності позиційної векторіальної похибки, основною характеристикою якої являється коваріаційна матриця іiї складових.

Радіус стохастичної складової кругової безпечної області судна вибирається таким чином, щоб вірогідність попадання істинного місця судна в область була близькою до одиниці, при умові, що центр кругової безпечної області співпадає з обсервованим місцем судна. Іншими словами, кругова область 3 центром в обсервованій позиції повинна накривати істинне місце судна з вірогідністю близькою до одиниці. В роботі для виконання цієї умови складене інтегральне рівняння, в якому в якості невідомої змінної міститься радіус кругової безпечної області судна. Так як в рівнянні інтеграл є визначений, а радіус знаходиться в межі інтегрування, то інтегральне рівняння перетворено до виразу в вигляді суми, що дозволяє визначити величину радіусу методом послідовних наближень.

Показано, що радіус кругової безпечної області судна залежить від дисперсій складових позиційної векторіальної похибки, які визначаються масштабним параметром закону розподілу, а в разі розподілу позиційної векторіальної похибки по змішаному закону першого або другого типу на величину радіусу впливає також істотний параметр закону розподілу.

Ключові слова: безпечність судноводіння, безпечна область судна, позиційна векторіальна похибка.

\section{Постановка проблемы в общем виде и ее связь с важными научными или практическими задачами}

Для обеспечения безаварийного плавания судна используется безопасная область, в которой находится судно, причем в эту область не должны попадать другие объекты во избежание аварийных происшествий. При выборе безопасного курса уклонения судна учитывается радиус круговой безопасной области судна, размеры которой определяются законом распределения 
векториальной погрешности. Поэтому анализ зависимости курса уклонения судна для безопасного расхождения с целью в зависимости от закона распределения векториальной погрешности является актуальным вопросом исследования проблемы безопасности судовождения.

Анализ последних достижений и публикаций, в которых начато решение данной проблемы и выделение нерешенных ранее частей общей проблемы

Вопросу построения судовой безопасной области посвящены многие работы, причем стандартным описанием такой области является круг, в центре которого находится судно, а радиусом является предельно-допустимая дистанция сближения.

Однако в ряде работ предлагается другая форма судовой безопасной области риска столкновения. Так, в работе [1] в качестве критерия безопасности предлагается «область столкновения», под которой имеется в виду некоторая область по направлению движения судна.

В работе [2] область навигационной безопасности описана эллипсом, которая используется в автоматизированной радиолокационной системе. При этом кривая, которая ограничивает зону, описывается из центра тяжести судна радиусом переменной длины, который зависит от длины судна, его скорости и курсового угла радиуса.

Процедура расчета большой оси эллипса зоны навигационной безопасности, как «динамической длины судна» и малой оси эллипса, как «динамической ширины судна» для плавания в портовых водах предложена в работе [3], причем оба параметра зависят от длины и ширины судна, его скорости и протяженности тормозного пути. В работе [4] рассмотрены более десяти применяемых в настоящее время форм судового домена опасности (безопасной области судна).

Формулировка целей статьи (постановка задачи) Целью статьи является анализ влияния закона распределения векториальной погрешности судна на размеры его круговой безопасной области и на курс уклонения при расхождении с опасной целью.

\section{Изложение основного материала исследования с обоснованием полученных научных результатов}

В работе [5] для оценки опасной области различной формы предлагается определить граничные размеры области $D$, в которой препятствие находится в области с заданной вероятностью $P_{d}$, близкой к единице.

Поэтому размеры области $D$ заданной формы вычисляются путем решения уравнения:

$$
\iint_{D} f(x, y) d y d x=P_{d}
$$

где $f(x, y)$ - двумерная плотность распределения векториальной позиционной погрешности. 
Для описания случайных погрешностей навигационных измерений применяется распределение Гаусса, двумерная плотность которого имеет вид:

$$
f(x, y)=\frac{1}{2 \pi \sigma_{x} \sigma_{y}} \exp \left[-\left(\frac{x^{2}}{\sigma_{x}^{2}}+\frac{y^{2}}{\sigma_{y}^{2}}\right)\right],
$$

где $\sigma_{x}$ и $\sigma_{y}$ - средние квадратические отклонения векториальной погрешности соответственно по осям $x$ и $y$, причем $\sigma_{x}=\sqrt{D_{x}}$ и $\quad \sigma_{y}=\sqrt{D_{y}}$.

Поэтому выражение (1) принимает вид:

$$
\frac{1}{2 \pi \sigma_{x} \sigma_{y}} \int_{D} \exp \left[-\left(\frac{x^{2}}{\sigma_{x}^{2}}+\frac{y^{2}}{\sigma_{y}^{2}}\right)\right] d y d x=P_{d} \text {. }
$$

Очевидно, для решения выражения (2) следует использовать численные методы. Рассмотрим способ решения выражения (2) для случая, когда безопасная область судна имеет форму круга, причем судно находится в центре области. В этом случае выражение (2) записывается в виде:

$$
\frac{4}{2 \pi \sigma_{x} \sigma_{y}} \int_{0}^{R_{\mathrm{st}}} \int_{0}^{\sqrt{R_{\mathrm{st}}^{2}-x^{2}}} \exp \left[-\left(\frac{x^{2}}{\sigma_{x}{ }^{2}}+\frac{y^{2}}{\sigma_{y}{ }^{2}}\right)\right] d y d x=P_{d},
$$

или для численного интегрирования:

$$
\sum_{i=0}^{R_{\mathrm{st}}} \sum_{j=0}^{\sqrt{R_{\mathrm{st}}^{2}-i^{2}}} \exp \left[-\left(\frac{i^{2}}{\sigma_{x}^{2}}+\frac{j^{2}}{\sigma_{y}^{2}}\right)\right]=\frac{P_{d}}{A}
$$

где $A=\frac{4}{2 \pi \sigma_{x} \sigma_{y}}$.

В уравнении (3) необходимо найти значение $R_{\mathrm{st}}$, для чего следует применить метод последовательных приближений. Так как $P_{d} \approx 1$, то для нормального распределения начальное приближение для $R_{\mathrm{st}}$ следует выбрать немножко меньше $3 \sigma$, где $\sigma$ принимает значение меньшего из с.к.о. $\sigma_{x}$ и $\sigma_{y}$.

Если векториальная погрешность подчинена смешанному закону распределения первого типа, то ее плотность распределения выражается следующим образом [6]:

$$
f_{1}(x, y)=\frac{\mathrm{A}_{\mathrm{x}} \mathrm{A}_{\mathrm{y}}}{\left(x^{2} / 2+\alpha_{x}\right)^{n+1}\left(y^{2} / 2+\alpha_{y}\right)^{n+1}}, \quad(n \leq 6)
$$


где $\mathrm{A}_{\mathrm{x}}=\frac{2^{n} \alpha_{x}{ }^{n+\frac{1}{2}} n !}{\sqrt{2} \pi 1 \cdot 3 \cdot \cdot(2 n-1)}, \mathrm{A}_{\mathrm{y}}=\frac{2^{n} \alpha_{y}{ }^{n+\frac{1}{2}} n !}{\sqrt{2} \pi 1 \cdot 3 \cdot \cdot(2 n-1)}$.

Расчет определяющего параметра $R_{\mathrm{st}}$ для стохастической области круговой формы производится с помощью следующего выражения:

$$
\sum_{i=0}^{R} \sum_{j=0}^{\sqrt{R_{\mathrm{st}}^{2}-i^{2}}} \frac{1}{\left(i^{2} / 2+\alpha_{x}\right)^{n+1}\left(j^{2} / 2+\alpha_{y}\right)^{n+1}}=\frac{1}{4 \mathrm{~A}_{\mathrm{x}} \mathrm{A}_{\mathrm{y}}} P_{d} .
$$

В случае распределения векториальной погрешности по смешанному закону второго типа с плотностью [6]:

$$
f_{2}(x, y)=\frac{\mathrm{B}_{\mathrm{x}} \mathrm{B}_{\mathrm{y}}}{\left(x^{2} / 2+\alpha_{x}\right)^{n+3 / 2}\left(y^{2} / 2+\alpha_{y}\right)^{n+3 / 2}}, \quad(n \leq 5)
$$

где $\mathrm{B}_{\mathrm{x}}=\frac{1 \cdot 3 \cdot 5 \cdot(2 n+1) \alpha_{x}{ }^{n+1}}{\sqrt{2} 2^{n+1} n !}$ и $\mathrm{B}_{\mathrm{y}}=\frac{1 \cdot 3 \cdot 5 \cdot(2 n+1) \alpha_{y}{ }^{n+1}}{\sqrt{2} 2^{n+1} n !}$ расчет параметра $R_{\mathrm{st}}$ производится с помощью выражения:

$$
\sum_{i=0}^{R_{\text {st }}} \sum_{j=0}^{\sqrt{R_{\mathrm{st}}^{2}-i^{2}}} \frac{1}{\left(i^{2} / 2+\alpha_{x}\right)^{n+3 / 2}\left(j^{2} / 2+\alpha_{y}\right)^{n+3 / 2}}=\frac{1}{4 \mathrm{~B}_{\mathrm{x}} \mathrm{B}_{\mathrm{y}}} P_{d}
$$

После определения радиуса стохастической области $R_{\mathrm{st}}$ предельнодопустимая дистанция сближения судна с целью $\mathrm{d}_{\mathrm{d}}$ находится из выражения:

$$
\mathrm{d}_{\mathrm{d}}=R_{\mathrm{st}}+R_{\mathrm{det}},
$$

где $R_{\mathrm{det}}$ - радиус детерминированной области, зависящий от габаритов судна и других детерминированных факторов. Используя полученное значение $\mathrm{d}_{\mathrm{d}}$, безопасный относительный курс $\mathrm{K}_{\text {oty }}$ находится с помощью формулы [6]:

$$
\mathrm{K}_{\text {oty }}=\alpha \pm \arcsin \left(\mathrm{d}_{\mathrm{d}} / \mathrm{D}\right) \text {, }
$$

где $\alpha$ и $\mathrm{D}$ - соответственно пеленг и дистанция между судном и целью.

Искомое значение курса безопасного уклонения определяется по известной зависимости [6]:

$$
\mathrm{K}_{\mathrm{y}}=\mathrm{K}_{\mathrm{oty}}+\arcsin \left[\rho^{-1} \sin \left(\mathrm{K}_{\text {oty }}-\mathrm{K}_{\mathrm{c}}\right)\right] \text {, }
$$

где $\rho=\mathrm{V}_{1} / \mathrm{V}_{2}$ и $\mathrm{V}_{1}, \mathrm{~V}_{2}$ - соответственно скорости судна и цели. 
Выводы и перспектива дальнейшей работы по данному направлению

Таким образом, в статье проведен анализ влияния закона распределения позиционной векториальной погрешности судна на размеры его круговой безопасной области, которая определяет курс уклонения судна при расхождении с целью в ситуации опасного сближения.

\section{ЛИТЕРАТУРА}

1. Lamb W. G. P. Calculation of the geometry of ship collision zones / Lamb W. G. P. // The Journal of Navigation. - 1989. - 42, № 2. - P. 298 - 305.

2. Goodwin E. M. A Statistical Study of Ship Domains / Goodwin E. M. // The Journal of Navigation. - 1975. - 28, № 3. - P. 328 - 341.

3. Погосов С.Г. Береговые системы управления движением судов/ Погосов С.Г., Москвин Г.И. - М.: Судовождение и связь, 1976. -54 с.

4. Вагущенко Л.Л. Расхождение с судами смещением на параллельную линию пути / Вагущенко Л.Л. - Одесса: Феникс, 2013. - 180 с.

5. Якушев А. О. Выбор оптимальной формы судовой безопасной зоны/ Якушев А. О. // Судовождение: Сб. научн. трудов./ ОНМА, Вып. 23. Одесса: «ИздатИнформ», 2013 - С.157-162.

6. Бурмака И.А. Управление судами в ситуации опасного сближения / И.А Бурмака., Э.Н Пятаков., А.Ю. Булгаков - LAP LAMBERT Academic Publishing, - Саарбрюккен (Германия), - 2016. - 585 с. 